\title{
The very elderly followed at a nephrology center: metabolic acidosis as a predictor of progressive chronic kidney disease
}

\author{
Marina Reis (D), Catarina Almeida, Catarina Ribeiro, Daniela Alferes, Ana Marta Gomes, João Carlos Fernandes \\ Nephrology Department, Centro Hospitalar Vila Nova de Gaia/Espinho, Vila Nova de Gaia, Portugal
}

\section{ABSTRACT}

Chronic kidney disease is an increasingly common diagnosis in the very elderly and identifying the patients who benefit from a nephrologist's intervention and the ones who would not might avoid wasteful or harmful interventions. The aim of this study is to identify the risk factors for progressive versus non-progressive chronic kidney disease in a population aged over 80 years old.

We performed a cohort single-center retrospective study including 101 patients over 80 years old with chronic kidney disease diagnosed for at least five years and followed regularly by a nephrologist. Progressive disease was defined as glomerular filtration rate declines greater than $5 \mathrm{~mL} / \mathrm{min} / 1.73 \mathrm{~m}^{2} /$ year.

Of the 101 patients, 33.7\% had progressive chronic kidney disease. The median glomerular filtration progression rate was 3.0 [2.1-6.0] $\mathrm{mL} /$ $\mathrm{min} / 1.73 \mathrm{~m}^{2} /$ year. Hypertension and diabetes mellitus prevalence was similar between groups. Nephrology follow-up time was longer in the progressive group ( 5.0 vs 2.0 years, $p=0.01$ ).

Regarding chronic kidney disease complications, $37.6 \%$ had anemia and half of these needed erythropoiesis-stimulating agents. None of the patients had hyperphosphatemia. About $18.8 \%$ presented metabolic acidosis. In multivariable analysis, after adjusting for covariables such as age, hypertension, and diabetes mellitus only the presence of metabolic acidosis (OR 0.4, Cl: 0.1-0.8) was associated with the development of progressive chronic kidney disease. Progressive chronic kidney disease group presented higher mortality (log rank 4.5, p=0.03). Ischemic cardiomyopathy (OR: 0.5, $\mathrm{Cl}: 0.2-0.9$ ) and progressive chronic kidney disease (OR: 0.6, Cl:0.3-0.8) were associated with all-cause mortality.

Our results showed that most elderly patients have non-progressive chronic kidney disease. Patients with metabolic acidosis seem to be at an increased risk for developing progressive disease. Most elderly patients die before reaching end-stage kidney disease, so it is important to look at progressive kidney disease in those patients as an important marker of comorbidity and privilege cardioprotective measures.

Keywords: acidosis; aging; chronic kidney disease.

C 2021 Portuguese Journal of Nephrology \& Hypertension. Published by Publicações Ciência \& Vida This is an open access article under the CC BY-NC-ND license (http://creativecommons.org/licenses/by-nc-nd/4.0/).

\section{INTRODUCTION}

Chronic kidney disease (CKD) diagnosis is increasingly common in the elderly. ${ }^{1-3}$ According to the Kidney Disease Improving Global Outcomes (KDIGO) 2012 guidelines, CKD is defined as a glomerular filtration rate (GFR) less than $60 \mathrm{ml} / \mathrm{min} / 1.73 \mathrm{~m}^{2}$ for three months or longer. ${ }^{4} \mathrm{~A}$ study performed among 610 individuals older than 70 years demonstrated that half had a measured GFR less than $60 \mathrm{~mL} / \mathrm{min} / 1.73 \mathrm{~m}^{2}$ and would therefore meet criteria for the diagnosis of CKD. ${ }^{5}$ On one hand, this could be explained by the increased prevalence of risk factors for CKD such as hypertension and diabetes with age. ${ }^{6}$ On the other hand, it is known that after 30 years, GFR decreases progressively as a consequence of renal cells senescence. ${ }^{7-9}$ Cellular senescence can result from progressive telomere shortening consequence of aging or as a response to various pathophysiologic stressors. The accumulation of senescent cells that can no longer replicate has been implicated in the development of kidney fibrosis by secretion of pro-fibrotic and pro-inflammatory mediators leading to nephrosclerosis, arteriolar hyalinosis, and thus fewer functional glomeruli available. ${ }^{9-11}$ Distinguishing between the patients who have age-associated kidney changes from those who have superimposed CKD is challenging. However, it crucial to identify which elderly patients have progressive kidney disease and a higher probability of developing severe forms of CKD from those whose renal function remains stable over time. This study aims to characterize patients with CKD over 80 years followed at a nephrology center and identify predictors for progressive versus non progressive CKD.

\section{METHODS}

Retrospective data was collected on patients aged over 80 years old with CKD, followed regularly at a nephrology single-center. Exclusion criteria comprised CKD diagnosis for less than 5 years and the absence of at least an annual creatinine value between $1^{\text {st }}$ January 2014 and $31^{\text {st }}$ December 2019. Therefore, 101 elderly patients were included in the study.

The data were collected from patients' electronic files and included demographic information, kidney disease cause, and comorbid conditions 
such as hypertension (blood pression superior to $140 / 90 \mathrm{mmHg}$ or use of any anti-hypertensive drug), obesity (body mass index more than 30 $\left.\mathrm{kg} / \mathrm{m}^{2}\right)$, ischemic cardiomyopathy, and diabetes mellitus. Other metrics were the presence of CKD complications such as anemia - defined as hemoglobin levels less than $12.0 \mathrm{~g} / \mathrm{dL}$ in women and less than $13.0 \mathrm{~g} /$ $\mathrm{dL}$ in men - or the need for epoetin supplementation; metabolic acidosis was defined as serum bicarbonate less than $22 \mathrm{mmol} / \mathrm{L}$ or bicarbonate suplementation; hyperphosphatemia was defined as phosphorus levels superior to $5.5 \mathrm{mg} / \mathrm{dL}$ or the use of phosphate binders. Intact parathyroid hormone (iPTH) levels, and the need for vitamin D analogs was also analyzed. Proteinuria was assessed in a spot urine sample by urine protein-to-creatinine ratio and considered significant when over $1 \mathrm{~g} / \mathrm{g}$.

CKD progression was assessed by the mean annual rate of GFR decline over at least five years. GFR was calculated with the CKD-EPI formula. Patients with GFR declines equal to or higher than $5 \mathrm{~mL} /$ $\mathrm{min} / 1.73 \mathrm{~m}^{2} /$ year were considered as presenting progressive kidney disease, and patients with a GFR decline less than $5 \mathrm{~mL} / \mathrm{min} / 1.73 \mathrm{~m}^{2} /$ year were considered as presenting non-progressive kidney disease. Other endpoints of interest were progression to end-stage kidney disease (ESKD) or death from any cause through 31 $1^{\text {st }}$ December 2019.

\section{Statistical Analyses}

Categorical variables were presented as frequencies and percentages and were compared with the use of Fisher's exact test or the chi-square test as appropriate. In continuous variables, normal distribution was checked using the Shapiro-Wilk test or skewness and kurtosis. Since continuous variables presented skewed distributions, they were presented as medians and $25^{\text {th }}$ and $75^{\text {th }}$ percentile. The Mann-Whitney test was used for univariable analysis. Binary logistic regression was performed in multivariable analysis, adjusted to risk factors for CKD progression (age, gender, diabetes mellitus, hypertension, obesity, ischemic heart disease, metabolic acidosis, and proteinuria). Kaplan-Meier estimates were used to evaluate survival curves between the progressive and non-progressive groups. Multivariate Cox proportional hazards were used to determine risk factors for mortality and included patients' comorbidities (age, gender, diabetes mellitus, ischemic cardiomyopathy, hypertension, proteinuria, obesity, metabolic acidosis, anemia, iPTH, and progressive CKD).

All reported $p$-values are two-tailed, with a $p$ value less than 0.05 indicating statistical significance. Statistical analyses were performed by SPSS version 23.0 (SPSS Inc., Chicago, IL).

\section{RESULTS}

A total of 382 patients over 80 years were followed in our nephrology center. Of these, 101 were included in this study, and the follow-up was 4.2 [1.7-6.9] years.

The clinical characteristics of the population are summarized in Table I. More than one-third of patients presented CKD of unknown cause, and none of the patients had performed a kidney biopsy. Proteinuria over $1 \mathrm{~g} / \mathrm{g}$ was present in $28.7 \%$ of patients, and $48.5 \%$ were using angiotensin converting enzyme inhibitors or angiotensin receptor blockers. There was a high prevalence of comorbidities: hypertension

\section{Table I}

Demographic and clinical characteristics of the population.

\begin{tabular}{|c|c|}
\hline Population Characteristics & $\begin{array}{c}\text { Patients } \\
\mathrm{N}=101\end{array}$ \\
\hline Age, median [IQR] & $85.0[82-88]$ \\
\hline Male, $\mathrm{n} \%$ & 56 (55.4\%) \\
\hline \multicolumn{2}{|l|}{ CKD cause } \\
\hline Unknown, n\% & $36(35.6 \%)$ \\
\hline Diabetic Kidney Disease, n\% & $31(30.7 \%)$ \\
\hline Ischemic Nephropathy, n\% & $25(24.8 \%)$ \\
\hline Chronic glomerulonephritis, n\% & $2(2.0 \%)$ \\
\hline Others, $\mathrm{n} \%$ & $6(5.9 \%)$ \\
\hline \multicolumn{2}{|l|}{ CKD stage } \\
\hline $3 A, n \%$ & $5(5.0 \%)$ \\
\hline $3 B, n \%$ & $34(33.7 \%)$ \\
\hline $4, \mathrm{n} \%$ & $44(43.6 \%)$ \\
\hline $5, n \%$ & $18(17.8 \%)$ \\
\hline Proteinuria, $n \%$ & $29(28.7 \%)$ \\
\hline Obesity, n \% & $23(23.0 \%)$ \\
\hline Ischemic Cardiomyopathy, n \% & $24(23.8 \%)$ \\
\hline Diabetes Mellitus, n \% & $43(42.6 \%)$ \\
\hline Hypertension, n \% & 94 (93.1\%) \\
\hline Anemia, $n \%$ & $38(37.6 \%)$ \\
\hline Metabolic Acidosis, n \% & $19(18.8 \%)$ \\
\hline Hyperphosphatemia, n \% & $0(0.0 \%)$ \\
\hline iPTH, median [IQR] & $164.5[122.5-226.3]$ \\
\hline ACEi/ARB, $n \%$ & $49,(48.5 \%)$ \\
\hline
\end{tabular}

ACEi/ARB - angiotensin converting enzyme inhibitor/angiotensin receptor blocker; CKD - Chronic Kidney Disease; iPTH - intact parathyroid hormone; std - standard.

was present in $93.1 \%$, diabetes mellitus in $42.6 \%$, and ischemic cardiomyopathy in $23.8 \%$. Regarding CKD complications, anemia was present in $37.6 \%$ of patients, and of these, $50.0 \%$ needed erythropoiesis-stimulating agents. Metabolic acidosis was present in $18.8 \%$. None of the patients presented hyperphosphatemia, and none were medicated with phosphate binders. Median iPTH levels were 164.5 [122.5- 226.3]. Vitamin D analogs were used on $5.0 \%$ of the patients. The median rate of decline of GFR was 3.0 [2.1-6.0] $\mathrm{ml} / \mathrm{min} / 1.73 \mathrm{~m}^{2} /$ year.

The comparison between the progressive and non-progressive CKD groups is shown in Table II. The large majority (66.3\%) presented non-progressive kidney disease. Nephrology follow-up time was also different between groups as progressive kidney disease group presented a longer follow-up (5.0 vs 2.0, p=0.01). Concerning KDIGO CKD stages, there were no statistically significant differences between groups (Table II).

Compared to the non-progressive CKD patients, the progressive kidney disease ones were obese $(38.2 \%$ vs $14.9 \%, p=0.04)$ and presented more frequent metabolic acidosis ( $29.4 \%$ vs $13.4, p=0.01)$. Proteinuria was slightly more common in the progressive group, but without statistical significance ( $34.4 \%$ vs $28.6 \%, p=0.56$ ). After adjusting for other variables (age, gender, diabetes mellitus, hypertension, obesity, ischemic cardiomyopathy, metabolic acidosis, and proteinuria), only the presence of metabolic acidosis (OR: 0.4, $\mathrm{Cl}$ : 0.1-0.8) was a predictor of progressive kidney disease (Table III). 


\section{Table II}

Comparison between progressive and non-progressive CKD groups

\begin{tabular}{|c|c|c|c|}
\hline & $\begin{array}{c}\text { Progressive CKD } \\
\mathrm{n}=34, \mathbf{3 3 . 7 \%}\end{array}$ & $\begin{array}{c}\text { Non-progressive CKD } \\
\mathrm{n}=67,66.3 \%\end{array}$ & $p$ value \\
\hline Age, median [IQR] & $85.0[81.0-87.0]$ & $83.0[82.0-88.0]$ & 0.45 \\
\hline Male, $\mathrm{n} \%$ & $18(52.9 \%)$ & $38(56.7 \%)$ & 0.72 \\
\hline \multicolumn{4}{|l|}{ CKD cause } \\
\hline Unknown, n \% & $12(35.3 \%)$ & $24(35.8 \%)$ & 0.96 \\
\hline Diabetic Kidney Disease, n \% & $14(41.2 \%)$ & $29(43.3 \%)$ & 0.84 \\
\hline Ischemic Nephropathy, n \% & $8(23.5 \%)$ & $17(25.4 \%)$ & 0.84 \\
\hline Chronic glomerulonephritis, n \% & $1(2.9 \%)$ & $1(1.5 \%)$ & 0.62 \\
\hline \multicolumn{4}{|l|}{ CKD Stage } \\
\hline $3 A, n \%$ & $0(0.0 \%)$ & $5(7.5 \%)$ & 0.12 \\
\hline $3 B, n \%$ & $15(44.1 \%)$ & $19(28.4 \%)$ & 0.13 \\
\hline $4, n \%$ & $13(50.0 \%)$ & $31(46.3 \%)$ & 0.44 \\
\hline $5, n \%$ & $6(17.7 \%)$ & $12(17.9 \%)$ & 0.98 \\
\hline Proteinuria, n \% & $11(34.4 \%)$ & $18(28.6 \%)$ & 0.56 \\
\hline ACEi/ARB, $n$ \% & $17(50.0 \%)$ & $32(48.5 \%)$ & 0.89 \\
\hline Obesity, n \% & $13(38.2 \%)$ & $10(14.9 \%)$ & 0.04 \\
\hline Ischemic Cardiomyopathy, n \% & $8(23.5 \%)$ & $16(23.9 \%)$ & 0.97 \\
\hline Diabetes Mellitus, n \% & $14(41.2 \%)$ & $29(43.3 \%)$ & 0.84 \\
\hline Hypertension, n \% & $30(88.2 \%)$ & $64(95.5 \%)$ & 0.17 \\
\hline Anemia, n \% & $11(32.4 \%)$ & $27(40.9 \%)$ & 0.40 \\
\hline Epoetin, n \% & $7(20.6 \%)$ & $12(17.9 \%)$ & 0.56 \\
\hline Metabolic Acidosis, n \% & $10(29.4 \%)$ & $9(13.4 \%)$ & 0.01 \\
\hline iPTH, median [IQR] & 173.5 [110.8-208.3] & $146.0[126.3-228.5]$ & 0.85 \\
\hline Vitamin D analogs, n \% & $1(3.1 \%)$ & $4(6.1 \%)$ & 0.54 \\
\hline $\begin{array}{l}\text { Duration of nephrology follow- } \\
\text {-up (years), median [IQR] }\end{array}$ & $5.0[2.1-9.0]$ & $2.0[1.4-5.0]$ & 0.01 \\
\hline
\end{tabular}

ACEi/ARB - angiotensin converting enzyme inhibitor/angiotensin receptor blocker; CKD - chronic kidney disease, IQR - interquartile range, IPTH - intact parathyroid hormone

During the study follow-up, $46.5 \%$ of patients died and $17.8 \%$ reached ESKD (Figure 1). Of those, most patients (55.6\%) initiated hemodialysis and the remaining were on conservative care. Figure 2

\section{Figure 1}

Population outcomes after the study follow-up.

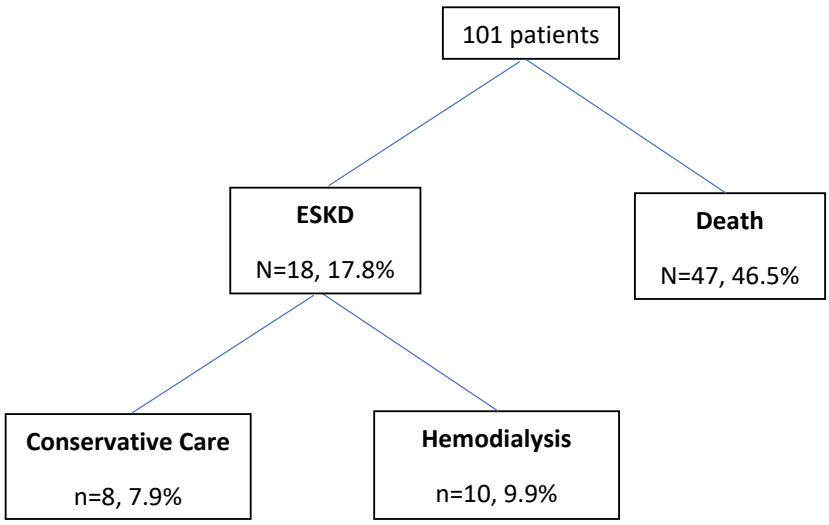

\section{$\underline{\text { Table III }}$}

Logistic regression for predictors of progressive CKD.

\begin{tabular}{l|c|c} 
& OR [IC 95\%] & p value \\
\hline Age & $0.9[0.8-1.1]$ & 0.37 \\
Gender & $1.1[0.3-3.4]$ & 0.90 \\
\hline Ischemic Cardiomyopathy & $1.5[0.4-5.7]$ & 0.60 \\
Diabetes Mellitus & $1.1[0.3-3.4]$ & 0.90 \\
Hypertension & $0.7[0.8-5.3]$ & 0.69 \\
Proteinuria & $0.6[0.1-2.2]$ & 0.40 \\
Obesity & $4.3[0.7-18.5]$ & 0.06 \\
Metabolic acidosis & $0.4[0.1-0.8]$ & 0.02
\end{tabular}

\section{$\underline{\text { Table IV }}$}

Survival analysis using Cox regression model.

\begin{tabular}{c|c|c} 
& HR [IC 95\% $]^{*}$ & p value \\
\hline Age & $1.0[0.9-1.1]$ & 0.76 \\
\hline Gender & $0.7[0.3-1.8]$ & 0.47 \\
\hline Diabetes Mellitus & $1.3[0.5-3.0]$ & 0.62 \\
\hline Ischemic Cardiomyopathy & $0.5[0.2-0.9]$ & 0.04 \\
\hline Hypertension & $0.9[0.2-3.4]$ & 0.89 \\
\hline Proteinuria & $1.1[0.4-3.0]$ & 0.93 \\
\hline Obesity & $2.3[0.8-6.3]$ & 0.11 \\
\hline Metabolic acidosis & $1.7[0.6-1.6]$ & 0.08 \\
\hline Anemia & $0.7[0.3-1.6]$ & 0.42 \\
\hline iPTH & $1.0[0.9-1.1]$ & 0.95 \\
\hline Progressive CKD & $0.6[0.3-0.8]$ & 0.03 \\
\hline
\end{tabular}

*HR were adjusted for age, gender, diabetes mellitus, ischemic cardiomyopathy, hypertension, metabolic acidosis, anemia, iPTH, proteinuria, and obesity.

\section{Figure 2}

Kaplan-Meier survival comparison between progressive and non-progressive CKD group.

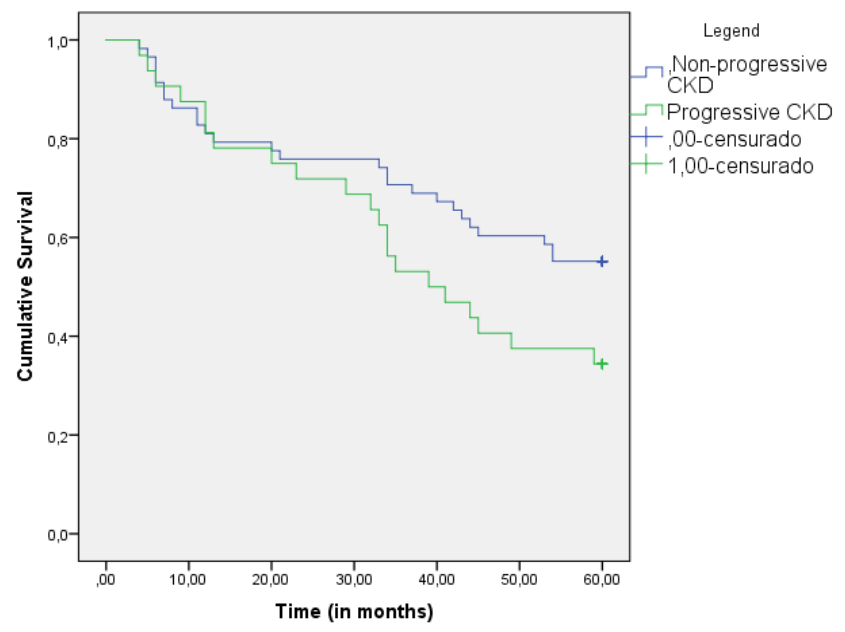


shows the Kaplan-Meier curves to compare survival between progressive and non-progressive CKD groups. The progressive CKD group had higher mortality (log rank 4.5, p=0.03). After adjusting for confounders, progressive kidney disease (OR 0.6, $\mathrm{Cl}: 0.3-0.8$ ) and ischemic cardiomyopathy (OR: $0.5, \mathrm{Cl}: 0.2-0.9)$ were risk factors for all-cause mortality (Table IV).

\section{DISCUSSION}

In our study, most of the very elderly patients presented non-progressive CKD and CKD cause was unknown in more than one-third. This is in line with other studies which show a slow GFR decline in the elderly. ${ }^{12-14}$ The slow GFR decline and the absence of an evident cause for CKD could be attributed to renal senescence, as has been reported. ${ }^{8,10}$ Diabetic kidney disease is currently the leading cause of CKD in developed countries ${ }^{15}$ and is the second-leading cause of CKD in this population. This could be a result of the competitive effects of death and progression to ESKD before the age of 80 years.

Our findings also suggest that metabolic acidosis is a predictor of progressive CKD. There is evidence that metabolic acidosis leads to CKD progression. ${ }^{16-19} \mathrm{~A}$ study with 134 patients with stage 4 CKD and metabolic acidosis randomized to either usual care or replacement with oral sodium bicarbonate for 2 years showed that patients receiving the supplementation had a significantly slower progression of CKD. ${ }^{18}$ A sub-analysis of the AASK trial suggested that serum bicarbonate level is an independent predictor of CKD progression and that higher serum bicarbonate levels within the normal range are associated with reduced hazard of mortality and CKD progression. ${ }^{19}$ In the kidney, metabolic acidosis is associated with an increase in aldosterone, endothelin, and angiotensin II levels. ${ }^{20-23}$ Endothelin-1 mediates increased renal acid excretion in response to a systemic acid challenge and pharmacological inhibition of endotelin-1 receptors using bosentan inhibited distal tubule acidification. ${ }^{21,22}$ Renin-angiotensin system activation is important for urinary acidification and also promotes proteinuria, kidney damage, and progressive CKD. ${ }^{23}$ Metabolic acidosis is also linked to protein-energy malnutrition and inflammation. ${ }^{24,25}$ Unfortunately, data regarding nutritional and inflammatory status was not available and therefore was not included on this study.

Proteinuria, is one of the most established factors for CKD progression 26,27 , was not statistically different between groups. We recognize that the use of the urine protein-to-creatinine ratio for proteinuria assessment in an elderly population with reduced muscle mass and common asymptomatic bacteriuria could induce some error.

Relative to other CKD complications, both groups were similar in terms of anemia, epoetin supplementation, and secondary hyperparathyroidism. It is relevant that none of the patients presented hyperphosphatemia. This could be caused by the decreased tubular reabsorption of phosphorus ${ }^{7}$ that occurs in elderly people, associated with the low dietary intake that is common in this population ${ }^{28}$.

Nephrology follow-up was longer in the progressive group. This is probably explained by the discharge of some patients with stable GFR, as opposed to patients with progressive GFR who are more likely to maintain nephrology follow-up. Also, patients with a faster GFR decline are more likely to be referenced to nephrology follow-up earlier.

During the study follow-up, considerably more patients died than progressed to ESKD. This is in line with other studies that reported that patients over 75 years old are more likely to die prior to progression to renal replacement terapies. ${ }^{29-31}$ In our cohort, the risk of death was superior in the progressive group and the risk of all-cause mortality was higher in patients with progressive CKD and in patients with ischemic cardiomyopathy. Therefore, progressive CKD in the elderly is likely to represent a pathological process and therefore it is a marker of multiple comorbidities and thus mortality. ${ }^{30}$

This study has some limitations that should be pointed out. First, it is a single-center retrospective study with a relatively small population and with the already known limitations related to the use of creatinine-based formulas to calculate GFR and protein-to-creatinine ratio to assess proteinuria in the elderly.

Our results showed that most elderly patients have non-progressive chronic kidney disease, and that metabolic acidosis seems to be a predictor of progressive disease. Therefore, metabolic acidosis, something that is sometimes overlooked when assessing CKD comorbidities, should be carefully assessed in elderly patients. Since metabolic acidosis is linked to nutrition and inflammation, more studies are needed to address the impact of these factors on CKD progression. Given that most patients died prior to progressing to ESKD, it is important to look at CKD in the elderly as an important marker of comorbidity. Beyond progressive CKD, ischemic cardiomyopathy is an important risk factor for all-cause mortality. These results emphasize the importance of the implementation of cardiovascular protection measures in those patients.

\section{Disclosure of potential conflicts of interest: none declared.}

\section{References}

1. Ji A, Pan C, Wang H, et al. Prevalence and associated risk factors of chronic kidney disease in an elderly population from eastern China. Int J Environ Res Public Health 2019;16(22):4383.

2. Amaral TLM, Amaral CA, Vasconcellos MTL, Monteiro GTR, Prevalence and factors associated to chronic kidney disease in older adults. Rev Saúde Publica 2019; 53:44.

3. Fukuma S, Ikenoue T, Shimizu S, et al. BiDANE: Big data analysis of medical care for the older in Kyoto: quality of care in chronic kidney disease and incidence of end-stage renal disease in older patients: A cohort study. Med Care 2020;58(7):625-31.

4. Kidney Disease: Improving Global Outcomes (KDIGO) CKD Work Group. KDIGO 2012 clinical practice guideline for the evaluation and management of chronic kidney disease: Kidney International Supplement 2013; 1-150.

5. Schaeffner ES, Ebert N, Delanaye $\mathrm{P}$, et al. Two novel equations to estimate kidney function in persons aged 70 years or older. Ann Intern Med 2012; 157(7):471-81.

6. Ferrannini E, Cushman WC. Diabetes and hypertension: the bad companions. Lancet 2012; 380(9841):601-10.

7. Musso CG, Oreopoulos DG. Aging and physiological changes of the kidneys including changes in glomerular filtration rate. Nephron Physiol 2011; 119(1):1-5.

8. Denic A, Glassock RJ, Rule AD. structural and functional changes with the aging kidney. Adv Chronic Kidney Dis 2016;23(1):19-28.

9. Zhou B, Wan Y, Chen R, et al. The emerging role of cellular senescence in renal diseases. J Cell Mol Med 2020; 24(3):2087-97.

10. Docherty MH, O'Sullivan ED, Bonventre JV, Ferenbach DA. Cellular senescence in the kidney. J Am Soc Nephrol 2019; 30(5):726-36.

11. Knoppert SN, Valentijn FA, Nguyen TQ, Goldschmeding R, Falke LL. Cellular senescence and the kidney: potential therapeutic targets and tools. Front Pharmacol 2019; 10:770.

12. Hemmelgarn BR, Zhang J, Manns BJ, et al. Progression of kidney dysfunction in the community-dwelling elderly. Kidney Int 2006; 69(12):2155-61.

13. Arora P, Jalal K, Gupta A, Carter RL, Lohr JW. Progression of kidney disease in elderly stage 3 and 4 chronic kidney disease patients. Int Urol Nephrol 2017;49(6):1033-40. 
14. Locatelli F, Pozzoni P. Chronic kidney disease in the elderly: is it really a premise for overwhelming renal failure? Kidney Int 2006; 69(12):2118-20.

15. Koye DN, Magliano DJ, Nelson RG, Pavkov ME. The global epidemiology of diabetes and kidney disease. Adv Chronic Kidney Dis 2018; 25(2):121-32.

16. Drawz PE, Babineau DC, Rahman M. Metabolic complications in elderly adults with chronic kidney disease. J Am Geriatr Soc 2012; 60(2):310-5.

17. Witham MD, Lamb EJ. Should chronic metabolic acidosis be treated in older people with chronic kidney disease? Nephrol Dial Transplant 2016; 31(11):1796-802.

18. Brito-Ashurst I, Varagunam M, Raftery MJ, Yaqoob MM. Bicarbonate supplementation slows progression of CKD and improves nutritional status. J Am Soc Nephrol 2009; 20(9):2075-84.

19. Raphael KL, Wei G, Baird BC, Greene T, Beddhu S. Higher serum bicarbonate levels within the normal range are associated with better survival and renal outcomes in African Americans. Kidney Int 2011; 79(3):356-62.

20. Wesson DE, Buysse JM, Bushinsky DA. Mechanisms of metabolic acidosis-induced kidney injury in chronic kidney disease. J Am Soc Nephrol 2020; 31(3):469-82.

21. Chen W, Abramowitz MK. Metabolic acidosis and the progression of chronic kidney disease. BMC Nephrol 2014; 15:55.

22. Wesson DE. Endogenous endothelins mediate increased distal tubule acidification induced by dietary acid in rats. J Clin Invest 1997, 99(9):2203-11.

23. $\mathrm{Ng} \mathrm{HY}$, Chen HC, Tsai YC, Yang YK, Lee CT. Activation of intrarenal renin-angiotensin system during metabolic acidosis. Am J Nephrol 2011; 34(1):55-63.

24. Dubey AK, Sahoo J, Vairappan B, Haridasan S, Parameswaran S, Priyamvada PS. Correction of metabolic acidosis improves muscle mass and renal function in chronic kidney disease stages 3 and 4: a randomized controlled trial. Nephrol Dial Transplant 2020; 35(1):121-9.

25. Zha Y, Qian Q. Protein nutrition and malnutrition in CKD and ESRD. Nutrients 2017, 9(3):208.

26. National Kidney Foundation. KDOQI clinical practice guideline for diabetes and CKD: 2012 Update. Am J Kidney Dis 2012; 60(5):850-86.
27. Tótoli C, Carvalho AB, Ammirati AL, Draibe SA, Canziani MEF. Associated factors related to chronic kidney disease progression in elderly patients. PLoS One 2019; 14(7):e0219956.

28. Lertdumrongluk P, Rhee CM, Park J, et al. Association of serum phosphorus concentration with mortality in elderly and nonelderly hemodialysis patients. J Ren Nutr 2013; 23(6):411-21.

29. Raman M, Green D, Middleton RJ, Kalra, PA. Comparing the impact of older age on outcome in chronic kidney disease of different etiologies: a prospective cohort study. J Nephrol 2018; 31:931-9.

30. Conway B, Webster A, Ramsay G, et al. Predicting mortality and uptake of renal replacement therapy in patients with stage 4 chronic kidney disease. Nephrology Dialysis Transplantation 2009; 24(6):1930-7.

31. Lundström UH, Gasparini A, Bellocco R, Qureshi AR, Carrero JJ, Evans M. Low renal replacement therapy incidence among slowly progressing elderly chronic kidney disease patients referred to nephrology care: an observational study. BMC Nephrol 2017; 18(1):59.

\section{ORCID}

Marina Reis (iD) 0000-0003-3061-3767

\section{Correspondence to:}

Marina Reis, MD

Nephrology Department, Centro Hospitalar Vila Nova de Gaia/Espinho

R. Conceição Fernandes; 4434-502 Vila Nova de Gaia, Portugal

E-mail: marina.reis9@gmail.com 